\title{
Patrones, competencias y desarrollo del pensamiento matemático
}

\author{
Patterns, skills and development of \\ mathematical thinking
}

\author{
Pastor Umanzor \\ Docente de Ciencias Matemáticas en e I CURSPS-UPNFM \\ pastoru2001@yahoo.com, pastor.umanzor@ gmail.com
}

\section{Resumen}

Este estudio supone que el desarrollo del pensamiento matemático es la condición de entrada y salida en el aprender matemáticas desde patrones matemáticos que derivan competencias matemáticas (comprensión, razonamiento, aprendizaje y cognición). Y es la oportunidad de aprender a enseñar para enseñar a aprender matemáticas. Estos conceptos le dan sentido a esta investigación, especialmente porque se desar rolla con la modalidad de la investigación cualitativa: la teoría fundamentada (Trinidad, C arrero y Soriano, 2006). Los resultados orientan a pensar que los estudiantes entrevistados del curso de matemática general del primer período del 2014 del CURSPS-UPNFM - son respetuosos en el aprender matemáticas, independientemente del uso de los patrones matemáticos y de las competencias matemáticas pero que revelan una oportunidad de aprender matemáticas y enseñar matemáticas si se les conduce a ese propósito. 
Pastor Umanzor

Palabras clave: patrones, competencias, pensamiento matemático, aprender a enseñar, enseñar a aprender, enseñar matemáticas.

\section{Abstrac}

Into this study the development of mathematical thinking is considered as the main condition of input and output in math learning from math patterns that generates math skills (comprehension, reasoning, learning and cognition). It is an opportunity of learning to teach how teaching to learn math. These concepts give meaning to this research, especially due it is developed with the method of qualitative research: grounded theory (Trinidad, C arrero and Soriano, 2006). The results suggest that students interviewed -from general mathematics course of the first period of 2014 to the CURSPS-UPN FM- are respectful into math learning, independently of the use of math patterns and skills, it which expose an opportunity to learn and to teach math into this purpose.

Keywords math patterns, math skills, math thinking, learning to teach, teaching to learn, teach math

\section{Introducción}

Este estudio representa una oportunidad de información para que profesores y estudiantes puedan reflexionar alrededor de la formación de profesores de matemáticas, pues en el primer período del 2014 inicia un curso específico de matemática general y al que se le puede dar seguimiento mediante la identificación de patrones matemáticos desde los que haya un acercamiento a competencias matemáticas que determinan el desarrollo del pensamiento matemático de seis estudiantes. Esto ayuda a redimensionar la formación de docentes en la enseñanza de las matemáticas. 
Patrones, competencias y desarrollo del pensamiento matemático

\section{El objeto de estudio}

En la experiencia profesional del suscrito, desde 1999 en San Pedro Sula, en la enseñanza de los cursos: Méto dos yTécnicas de Investigación Educativa, Metodología de Investigación Cuantitativa, Metodología de Investigación Cualitativa, Seminarios de Investigación I y II, Didáctica de las Matemáticas, Diseño y Desarrollo Curricular, Evaluación; Matemáticas, Pre-cálculo, Álgebra, G eometría, y Vectores y Matrices, como aporte en la formación de docentes de matemáticas y otras áreas, no se visibilizan en los estudiantes de la carrera de matemáticas conceptos claros sobre el pensamiento matemático venidos del aprender matemáticas en los niveles precedentes.

\section{El problema: ¿Q ué patrones y competencias están presentes en el pensamiento matemático de los estudiantes que ingresaron al CURSPS-UPNFM en febrero del 2014, a la carrera de matemáticas?}

\section{Los objetivos de la investigación}

0 bjetivo general

Comprender cómo los patrones y competencias están presentes en el pensamiento matemático de los estudiantes que ingresar on al CURSPSUPN FM en febrero del 2014, a la C arrera de Matemáticas.

O bjetivos específicos

1. Entender las creencias de los estudiantes respecto del ser profesores de matemáticas.

2. Inter pretar las tendencias de los patrones simbólicos, numéricos y geométricos entre los estudiantes de la cohorte del año 2014.

3. Categorizar las competencias matemáticas de los estudiantes: comprensión, razonamiento, aprendizaje y cognición asociados con el pensamiento matemático.

4. Establecer los alcances del pensamiento matemático de los 
Pastor Umanzor

estudiantes.

5. Construir una teoría que fundamente el proceso de aprender matemáticas a partir de los patrones matemáticos de los estudiantes evaluado s que pasa por las competencias y muestran un desarrollo claro del pensamiento matemático.

\section{Métodos y Materiales}

La investigación ha sido perfilada desde la teoría fundamentada (Trinidad, Carrero y Soriano, 2006) porque es una modalidad de investigación cualitativa que permite la construcción de una nueva teoría a partir de la recolección de datos en bruto, mismos que se organizan con el propósito de establecer la información que da sentido de respuesta a una situación particular, que interpretada produce un determinado conocimiento.

\section{El diseño de la investigación}

Este estudio se inició con la identificación de patrones matemáticos: simbólicos, numéricos, y geométricos en función de una prueba con tres ejercicios a fin de establecer las competencias matemáticas: comprensión, razonamiento, aprendizaje y cognición, que ayudarán a interpretar el desarrollo del pensamiento matemático de los estudiantes en función de la teoría fundamentada.

La teoría fundamentada puede definirse como "una aproximación que propone un método de análisis, que implica la utilización de un conjunto de técnicas sistemáticamente aplicadas" (Trinidad, C arrero y Soriano, 2006: 16). Con esta definición puede entenderse que su finalidad es la emergencia de una teoría procesada de manera inductiva y sobre un área del conocimiento específico. Se busca generar y hacer emerger un conocimiento del campo de estudio, mismo que debe estar fundamentado en lo sustantivo del campo y desarrollarse inductivamente. 
Esta investigación se desarrolló considerando tres fases: la delimitación del objeto de estudio, la realización del trabajo de campo y la triangulación teórica como técnica de análisis. En la primera fase se hizo una definición del marco conceptual y las cuestiones a abordar en la investigación. En la segunda, se elaboró una prueba con tres ejercicios orientados a mostrar la emergencia de patrones matemáticos; luego, después de aplicada la prueba y revisar los resultados, se seleccionó un grupo de seis estudiantes (con respuesta en los extremos: bueno, más o meno s bueno y deficiente), para establecer las tendencias de los patrones matemáticos.

Cada estudiante se entrevistó por separado con la idea de no contaminar las respuestas y asegurar la saturación de los conceptos, según la definición del muestreo teórico. C ada entrevista fue transcrita por el investigador para asegurar sistematicidad en la determinación de las tendencias de los patrones y las competencias matemáticas que le darían sentido al desarrollo del pensamiento matemático expresado.

La tercera fase fue el momento de la construcción de la teoría resultante en la investigación a partir de los datos recolectados en el campo con base en las catego rías, subcatego rías y códigos. Se hicieron las respectivas y sucesivas comparaciones entre los patrones, las competencias matemáticas expresadas por los estudiantes, tanto en la resolución de ejercicios como en la entrevista, para darle sentido al pensamiento matemático expresado por ellos.

En esta fase se consideraron el desarrollo de tres perspectivas (siguiendo los alcances de la investigación en la teoría fundamentada): una analítica, referida al análisis de los discursos de los estudiantes y una codificación selectiva; una interpretativa, relacionada con la detección de la categoría central y el proceso de construcción de conocimientos por los estudiantes, la integración teórica generada de los datos en bruto y sistematizada en una teoría emergente en comparación con la teoría formal. Por último, la redacción del informe preliminar, para su devolución y después se construyó el informe final. 
Pastor Umanzor

\section{Categorías de análisis}

El estudio asume como categorías de análisis a los seis estudiantes, a quienes se les consultó sobre los alcances del desarrollo de su pensamiento matemático (categoría central) desde la resolución de ejercicios con base en patrones matemáticos (subcategoría base) de los que se derivaron las competencias matemáticas específicas (subcategoría intermedia).

\section{Participantes}

La Sección Académica de Matemáticas del CURSPS-U PN FM decidió tomar un primer curso de matemáticas general con inicio en el primer período del 2014 hasta finales del 2017, como un curso especial en la C arrera, para garantizar diferencias en la formación de profesores de matemáticas. El curso se organizó con 54 estudiantes pero la prueba se aplicó a 44, para seleccionar 6 de ellos. Por tanto, los resultados del estudio se basan en esos seis estudiantes entrevistados y codificados como: estudiantes E1, E3, E4, E5, E20 y E29.

\section{Instrumentos de recolección de datos:}

Para el desarrollo de esta investigación se utilizaron dos instrumentos: una prueba de conocimientos matemáticos de los estudiantes basada en patrones matemáticos (simbólicos, numéricos y geométricos).Y una guía de entrevista relacionada con los objetivos de la investigación. Los ejercicios fueron:

Tabla 1: La prueba consta de tres ejercicios

1) Siguiendo el patrón de las 3 primeras figuras, ¿Cuantos triángulos pequeños aparecerán en la sexta figura?
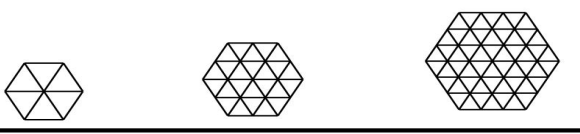

2) Decimos que un número es ascendente si cada una de sus cifras de la derecha es mayor que la de su izquierda ¿Cuántos números ascendentes hay entre 4000 y 5000 ? 
3) Las aristas de un cubo de madera miden $3 \mathrm{~cm}$ cada una. D os de sus caras opuestas se pintan de rojo y las otras cuatro se pintan de azul. El cubo se pica en 27 cubitos iguales de 1 de volumen. Determine: ¿C uántos de esos cubitos tienen tres de sus caras pintadas? ¿C uántos tendrán dos caras pintadas? ¿Cuántas tendrán una sola cara pintada?

Fuente: Construcción propia.

Con el primer ejercicio se pretendía que los estudiantes pudieran identificar los patrones 6 y el cuadrado de los 6 primeros dígitos, de manera que reflejaran lo simbólico, lo numérico y lo geométrico al mismo tiempo. Con el segundo, se esperaba que mostraran un acercamiento al concepto combinación sin repetición. Con el tercero, se esperaba que los estudiantes pudieran contar las caras pintadas 0 no del cubo "Rubik".

Se escogieron estos ejercicios porque se buscaba la emersión de las preguntas que direccionaran la investigación: ¿Q ué experiencias tenían los estudiantes para la identificación de patrones matemáticos? ¿D esde qué lógicas los estudiantes responden a las pruebas? ¿Cómo operan los estudiantes la solución a determinados problemas? ¿Cómo las operatorias reflejan sistematicidad en las respuestas a las preguntas?, ¿Cómo la resolución de los problemas se ajusta a la interpretación de las competencias matemáticas como comprensión, razonamiento, aprendizaje y cognición? Y ¿cómo estructuran un pensamiento matemático propio de los problemas planteados?

\section{Técnicas de análisis de datos}

La misma modalidad de investigación teoría fundamentada define un método de análisis comparativo constante, para la construcción de una nueva teo ría emergente. En tal caso, las técnicas de análisis se asociaron al uso de estrategias desarrolladas por los estudiantes en la solución de los ejercicios y explicadas en las entrevistas; en función de ellas se crearon matrices que permitieran comparar los patrones identificados en sus respuestas, su interpretación y establecer, a partir de la rúbrica 
Pastor Umanzor

expresada en las instrucciones, la comprensión del fenómeno en estudio: el desarrollo de su pensamiento matemático.

\section{Discusiónteórica}

Esta investigación ofrece espacios para revelar las potencialidades de los estudiantes entrevistados respecto de aprender matemáticas y ser docentes, considerando que es un grupo especial que se familiarizará con el problema, la búsqueda de estrategias, la aplicación de una y su verificación, tal como lo definió Polya (1987).

Cada estudiante, desde su lógica, se muestra comprometido con aprender matemáticas y ser profesor de matemáticas, por tanto, espera que en el desarrollo de las clases de matemáticas haya un acercamiento a la construcción de conocimientos sustantivos, sintácticos y sobre to do didáctico sen el CURSPS-UPN FM. Es una aspiración válida.A hora, quedan preguntas por contestar: ¿Q ué se hará en la Carrera de Matemáticas para satisfacer esas inquietudes profesionales de estos estudiantes? ¿C uáles serán los cambios en la enseñanza y la evaluación en competencias? Si el enfoque que se desarrolla en el CURSPSUPN FM es el de la formación basada en competencias: ¿Cómo se reflejará en el aula esa condicionante? ¿Q ué diferencias hay con la evaluación por competencias?

\section{Patrones matemáticos}

El sentir y pensar de los estudiantes entrevistados está conectado con el aprender matemáticas a partir de la identificación de patrones matemáticos, las competencias matemáticas y el desarrollo del pensamiento matemático, que es una discusión planteada en el mundo expresada en qué fundamentos matemáticos se deben enseñar y cómo enseñarlos. También, sobre el equilibrio de las prioridades de los diferentes temas. Estas discusiones entre matemáticos ayudan a fortalecer los cambios que deben darse en la sociedad, en la tecnolo gía, en las escuelas considerando el pensamiento matemático del próximo siglo. Siguiendo a Steen (2004: 15): “Los humanos utilizan el lenguaje 
Patrones, competencias y desarrollo del pensamiento matemático

de la matemática para describir patrones".

Estar al servicio de la humanidad implica, también, que el profesor de matemática sea capaz de transformar el saber sabio en el saber a enseñar (C hevallard, 2000) en el ámbito familiar, la educación prebásica, básica, media y superior. Según Senechal (2004: 149): "Los patrones son evidentes en la simple repetición de un sonido, de un movimiento 0 de una figura geométrica". Esto dice que los patrones son un elemento importante en la enseñanza de las matemáticas, por tanto, en la formación de profesores de matemáticas.

\section{Competencias matemáticas}

Esto sugiere una pregunta: ¿cómo aprendió a enseñar el profesor y cómo enseña matemáticas? Pregunta que aún hay que buscarla en la investigación en educación matemática, en la didáctica de las matemáticas, y en la transposición didáctica en las aulas, especialmente porque se asume que los profesores saben matemáticas pero falta saber cómo hacen para transformar los contenidos matemáticos que sean enseñables y que traigan como consecuencia el aprendizaje de leyes, normas reglas, principios de las matemáticas y no solamente la operatoria, es decir, el desarrollo del pensamiento matemático.

En tal virtud, se supone que el desarrollo de las competencias matemáticas pasa por la estructuración de la comprensión de lo leído, el desarrollo de un razonamiento lógico que contribuya a un aprendizaje general y específico de las ciencias matemáticas, porque siguiendo a N agusia (2014: 10): "Las matemáticas son un conjunto de saberes y de prácticas asociados, en una primera aproximación, al uso reflexivo de los números y de las formas, y de los lenguajes que se van progresivamente completando hasta constituir un modo valioso de analizar situaciones variadas".

Si este es el valor de las matemáticas, las competencias matemáticas asumen un papel protagónico en la construcción del pensamiento matemático. Siguiendo a N agusia (2014: 12): "La competencia 
matemática consiste en la habilidad para utilizar y relacionar los números, sus operaciones básicas, los símbolos y las formas de expresión y razonamiento matemático,...". El desarrollo de la competencia matemática supone aplicar aquellas destrezas y actitudes que permiten razonar matemáticamente, comprender una argumentación matemática y expresarse y comunicarse en el lenguaje matemático.

\section{Pensamiento matemático}

No existe una clara definición de pensamiento matemático pero se infiere de lo que los psicólogos llaman pensamiento. Castro Martínez (1993: 29) citando a Meyer (1986) parte de tres ideas sobre el concepto: 1) el pensamiento es cognitivo, 2) establece un conjunto de operaciones sobre el conocimiento, 3) es dirigido y tiene como resultado la resolución de problemas.

En dirección a la construcción del pensamiento matemático existen aristas que ayudan a configurar una propuesta de precisión en el siguiente sentido, considerando la propuesta de Cantoral (2002:3) "el pensamiento matemático incluye, por un lado, pensamiento sobre tópicos matemáticos y, por otro, procesos del pensamiento avanzados, como abstracción, justificación, visualización, estimación 0 razonamiento mediante hipótesis". Por tanto, los profesores de matemáticas estarían haciendo un bien a la humanidad si en el proceso de formación de docentes, los estudiantes logran desarrollar el pensamiento matemático.

\section{El pensamiento matemático y el aprender a enseñar}

Si la idea del desarrollo del pensamiento matemático es reciente y requiere mucha investigación, entonces, se esperaría que su relación con el aprender a enseñar para enseñar a aprender matemáticas sea mucho más nueva. Siguiendo a Cantoral (2002: 7): "N uestro objetivo es explorar el sentido que tiene el desarrollo del pensamiento matemático entre los estudiantes en el transcurso de la enseñanza". 
Es la oportunidad de construcción de una nueva imagen en el ideario de un profesor que está dispuesto a aprender a desaprender todo aquello que implica un compromiso con el cambio para mejorar, mejorar el vestir, la paciencia, la sapiencia, el ser docente que forma docentes; actividades que lo harán toda su vida.

\section{Resultados}

En este apartado se incluyen los resultados del trabajo de campo y la interpretación que de él emerge. Las instrucciones eran la rúbrica que ayudara en la resolución de los problemas, asi:" Instrucciones: Resuelva cada ejercicio detallando los datos que se le dan y lo que le piden; exprese una estrategia que usted utilizaría para resolver cada ejercicio; ejecute la estrategia identificada y verifique su resultado. Al final explique cómo encontró el resultado".

En lo que sigue, se presentan tres ejemplos, uno por ejercicio, de un mismo estudiante -el que los desarrolló casi completamente-, como ejemplo de las estrategias utilizadas en la resolución de cada problema. Falta espacio para ubicar todas las estrategias utilizadas por los seis estudiantes de la muestra, sin embargo, se toman sus explicaciones como la información confirmada en la investigación. Estos ejemplos son del Estudiante E29.

Ejercicio 1

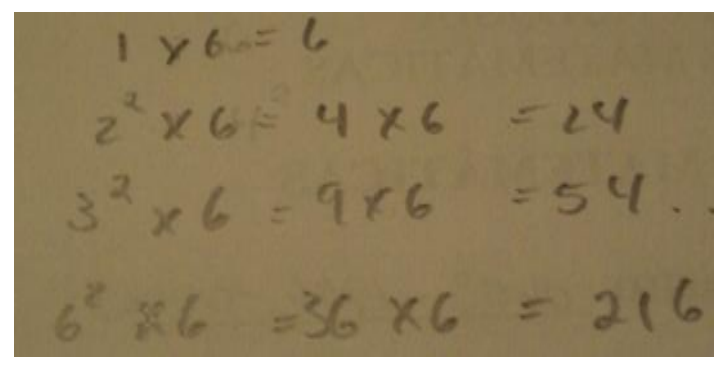

Paradigma - Revista de Investigación Educativa. Año 21. No. 35 


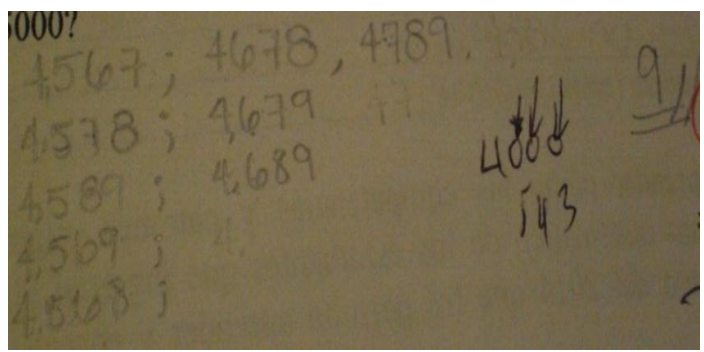

Ejercicio 2

Ejercicio 3

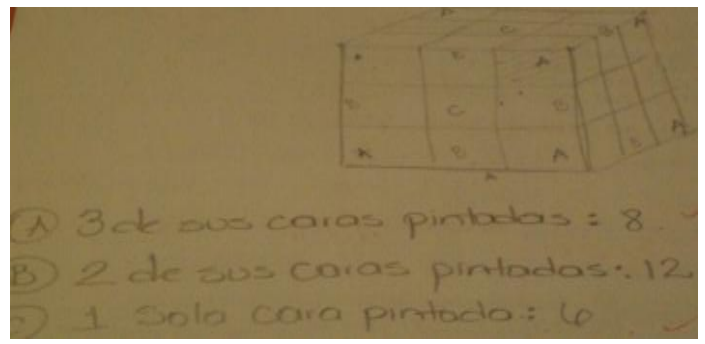

Una mirada rápida a las estrategias utilizadas por este estudiante a cada ejercicio indica que: en el ejercicio 1 tenía clara la idea del patrón numérico específico; en el ejercicio 2 pudo identificar nueve números que ayudaron en la identificación del patrón, pero no lo logró. En el ejercicio 3 identificó la cantidad de caras pintadas pero no la no pintada. En todo caso, este estudiante tiene un pensamiento claro, fue el mejor resultado de los seis.

Esta perspectiva de la resolución de los problemas propuestos hace suponer que es posible un encuentro con patrones matemáticos, competencias matemáticas y desarrollo del pensamiento matemático en los tres ejercicios y en cada una de las respuestas de los seis estudiantes entrevistados, que es lo que se muestra en los siguientes testimonios:

\section{Identificación de patrones matemáticosy la derivación de las competencias:}

A continuación se presentan los testimonios de los estudiantes entrevistados desde los cuales se identifican los patrones matemáticos. 
Patrones, competencias y desarrollo del pensamiento matemático

Luego, las competencias matemáticas derivadas de los patrones identificados.

Matriz: Comparación de los ejercicios con los estudiantes en su discurso para la identificación de patrones

\begin{tabular}{|c|c|c|c|}
\hline $\begin{array}{l}\text { EJERCICIOS } \\
\text { ESTUDIANTES }\end{array}$ & 1 & 2 & 3 \\
\hline E1 & $\begin{array}{l}\text { “de allí yo me } \\
\text { imaginé que poco a } \\
\text { poco iba ir } \\
\text { partiendo la pizza } \\
\text { en más pedazos y } \\
\text { después lo que } \\
\text { hice fue dibujar una } \\
\text { recta numérica y } \\
\text { puse los pedazos de } \\
\text { la primera } \\
\text { pizza,...". }\end{array}$ & $\begin{array}{l}\text { "Este ejercicio sé } \\
\text { que no lo hice bien, } \\
\text { verdad". }\end{array}$ & $\begin{array}{l}\text { "la verdad es que } \\
\text { me centralicétanto } \\
\text { en el primer } \\
\text { ejercicio". }\end{array}$ \\
\hline E3 & $\begin{array}{l}\text { "Tenemos esta } \\
\text { figura y tiene } 6 \\
\text { triángulos, aquí } \\
\text { aumentó el tamaño } \\
\text { de la siguiente } \\
\text { figura, entonces } \\
\text { cada triángulo se } \\
\text { dividió en } 4 ; \ldots, \\
\text { entonces } 6 \times 4= \\
24 \text { ". }\end{array}$ & $\begin{array}{l}\text { "yo entendía que } \\
\text { me pedía el número } \\
\text { de la izquierda y yo } \\
\text { quedé viendo a la } \\
\text { izquierda de 4". }\end{array}$ & $\begin{array}{l}\text { "no sabía, no } \\
\text { estaba segura de } \\
\text { cuántos cubitos } \\
\text { habían al final". }\end{array}$ \\
\hline E4 & $\begin{array}{l}\text { "O bservé un } \\
\text { patrón". "Ya estaba } \\
\text { seguro de mi idea". }\end{array}$ & $\begin{array}{l}\text { "no le entendí, no } \\
\text { tenía ni la más } \\
\text { mínima idea". }\end{array}$ & $\begin{array}{l}\text { "El cubo me sirvió } \\
\text { sólo para plantear } \\
\text { el problema, } \\
\text { entonces, lo hice } \\
\text { mental". }\end{array}$ \\
\hline E5 & $\begin{array}{l}\text { “entonces, mi } \\
\text { análisis fue así: el } \\
\text { primero tiene } 6 \text {, } \\
\text { sólo está formada } \\
\text { por un triángulo; el }\end{array}$ & $\begin{array}{l}\text { "Y lo único que me } \\
\text { dio la lógica, que no } \\
\text { me salió bien, irme } \\
\text { a los números de la } \\
\text { izquierda". }\end{array}$ & $\begin{array}{lr}\text { "uno no } & \text { está } \\
\text { preparado } & \text { para } \\
\text { responder } & \text { de } \\
\text { inmediato". } & \end{array}$ \\
\hline
\end{tabular}

Paradigma - Revista de Investigación Educativa. Año 21. No. 35 


\begin{tabular}{|c|c|c|c|}
\hline & $\begin{array}{l}\text { segundo, tenemos } \\
\text { que está formada } \\
\text { por } 4 \text { triángulos, } \\
\text { tenemos tres } \\
\text { abajo y uno arriba; }\end{array}$ & & \\
\hline E20 & $\begin{array}{l}\text { "Yo pensaba en } \\
\text { esto, verdad, } 6 \\
\text { elevado a la } 2 \text { era } \\
12 " .\end{array}$ & $\begin{array}{l}\text { "No, no lo } \\
\text { entendí". }\end{array}$ & $\begin{array}{l}\text { "Bueno, yo lo traté } \\
\text { de hacer, y bueno lo } \\
\text { sumé todo". }\end{array}$ \\
\hline E29 & $\begin{array}{l}\text { "Porque es el } \\
\text { patrón", "lo } \\
\text { primero que hice } \\
\text { fue colocar el } \\
\text { patrón, y por ende } \\
\text { sabía que } 3 \text { al } \\
\text { cuadrado es } 9 \times 6 \text { es } \\
54 \text { ". }\end{array}$ & $\begin{array}{l}\text { "me está diciendo } \\
\text { ascendente y los voy } \\
\text { a poner en orden y } \\
\text { ese número va } \\
\text { aumentando, me } \\
\text { imagino que los } \\
\text { dígitos van en forma } \\
\text { ascendente". }\end{array}$ & $\begin{array}{l}\text { "Creo que me } \\
\text { enfoqué más en el } \\
\text { rojo y en el azul". } \\
\text { "Faltó uno, y es el } \\
\text { que está en el } \\
\text { centro". }\end{array}$ \\
\hline
\end{tabular}

Fuente: Construcción propia

De lo expresado por el estudiante E1 se puede entender que puede resolver un problema matemático si lo dibuja primero como un slide de pizza por la forma triangular y es vista como una estrategia de solución. Hacer este ejercicio significó identificar que "el patrón va a ser 2 siempre" y la base 6, según la figura a la que debería llegar, la sexta. Con ello mostró las competencias esperadas. Pero no ocurrió lo mismo con los otros ejercicios. En consecuencia, no mostró el desarrollo de su pensamiento matemático.

El estudiante E3 informó que "ya lo había hecho en otro ejercicio que era parecido a este", sólo que aplicado a la "novena figura". Q uiso hacerlo igual y no logró el resultado, entonces no pudo mostrar las competencias esperadas. Tampoco con los otros dos ejercicios. Por tanto, no mostró el desarrollo de su pensamiento matemático.

El estudiante E4 de entrada afirmó haber encontrado un patrón: "O bservé un patrón". Esa era suficiente información para aceptar que 
había dominio de las competencias que lo llevaron a un claro pensamiento matemático. No entendió la definición de números ascendentes y si pudo contar las caras pintadas, aunque no la no pintada. Por efecto de haber encontrado los patrones de los ejercicios 1 y 3 se puede aceptar que mostró el desarrollo de su pensamiento matemático.

El estudiante E5 asumió que el patrón es una representación y eso se convierte en una aproximación a la comprensión y al razonamiento como competencias fundamentales en la búsqueda del desarrollo del pensamiento matemático; no dejó en claro haberlo logrado. Igual ocurrió con los otros dos ejercicios. Lo que lo deja fuera del contexto en el desarrollo de su pensamiento matemático.

El estudiante E20 llegó a un resultado correcto pero en función de una operación incorrecta: elevar al cuadrado un valor y luego multiplicarlo por el mismo cuadrado, $6^{2}$ debe ser 36 pero para él era 12. No entendió la definición de números ascendentes. Y tampoco contar las caras pintadas o no del cubo "Rubik". En consecuencia, no mostró el desarrollo de su pensamiento matemático.

Finalmente, no hay duda que el estudiante E29 tiene claramente definido un pensamiento matemático, pues de entrada identificó el patrón numérico lo que hace visible el do minio que tiene de las competencias matemáticas asociadas y, como consecuencia, un claro pensamiento matemático. Aunque no logró expresar el patrón de los números ascendentes, sí pudo encontrar nueve números de los 10, sin el patrón; pudo, también, contar las caras pintadas aunque no la cara pintada - lo aceptó en la entrevista-. Esto lo ubica como un estudiante que si ha logrado el desarrollo de su pensamiento matemático.

\section{Identificación del pensamiento matemático}

\section{Aspectos semejantes}

En función de los ejercicios 1 al 3 propuestos, los estudiantes E1, E3, E5 y E20 tenían idea de cómo resolver el primer problema, no así los 
Pastor Umanzor

otros dos. Estos estudiantes no mostraron un claro dominio en la identificación de los patrones matemáticos, aunque pudieran manifestar ciertas competencias matemáticas como la comprensión y razonamiento pero no las de aprendizaje y cognición, lo que queda claro que les falta un desarrollo del pensamiento matemático. En cambio, los estudiantes E4 y E29 hicieron visible el manejo de los patrones matemáticos que derivan en competencias matemáticas con lo que se garantiza el desarrollo del pensamiento matemático de ambos.

\section{Aspectos diferentes}

El estudiante E1 pudo "dibujar una recta numérica", unas gradas para simular una escalera; además, el uso de los trozos de pizza como lenguaje que muestra un pensamiento matemático claro, sin embargo, no logró entroncar los patrones simbólicos, numéricos y geométricos en el resultado final, en el ejercicio 1, no así en los otros dos problemas. El estudiante E3 dijo "quedé en blanco" y eso es determinante para establecer la no presencia de un patrón matemático, quizás comprende y razona en función de la operato ria matemática pero eso no garantiza el haber logrado desarrollar un claro pensamiento matemático.

El estudiante E4 tenía clara la idea de patrón matemático que derivan en competencias, lo que mostró un claro pensamiento matemático porque dijo "Yo estaba seguro de mi idea", en el primer y tercer ejercicio, no así en el segundo porque rondó en la explicación. Lo claro es que su seguridad e inseguridad lo posicionan en la frontera de acercarse al desarrollo del pensamiento matemático esperado en la UPN FM.

El estudiante E5 dijo "fui formando cada triángulo", "sumé los extremos de las columnas formadas y no me dio el resultado" pero en ningún caso obtuvo los resultados esperados. El estudiante E20 mostró una teoría novedosa porque dijo:"Yo pensaba en esto, verdad, 6 elevado a la 2 era 12", lo que parece incongruente que se entienda esta expresión numérica $6^{1}=6,6^{2}=12,6^{3}=18,6^{4}=24,6^{5}=30$ y $6^{6}=36$, en el primer 
ejercicio, aunque el resultado alcanzado era correcto. Pero en los otros dos ejercicios no logró entender ni los números ascendentes ni el conteo como concepto básico en los patrones matemáticos.

El estudiante E29 dijo "lo vi como un patrón", identificó nueve de los números ascendentes y dibujó las respuestas del tercer ejercicio. A unque no llegó a las respuestas correctas a los do últimos ejercicios, es visible considerar que conoce lo que es un patrón matemático, discierne sobre las competencias matemáticas con lo que se puede afirmar ha desarrollado un claro pensamiento matemático.

El pensamiento matemático y el aprender a enseñar

Este apartado se agrega porque se asume como condición básica que el estudiante que ha desarrollado un claro pensamiento matemático, puede aprender a enseñar para enseñar a aprender matemáticas y ser un formador de formadores durante su vida. La perspectiva detrás es que entre los estudiantes entrevistados hay unos que muestran una disposición personal y otro, una tradición familiar.

\section{Disposición personal}

E1: $\quad$ "Decidí estudiar en la UPN FM porque es que me llega por ser maestra", "entonces quise continuar con mis sueño s y la UPN FM se supone me ayudará a cumplirlo". "N o ser una maestra del montón sino una maestra de calidad". "Me gustan los retos y las matemáticas significan un reto para mí", "no soy muy buena pero mi intención es llegar".

E3: "me falta bastante, porque yo siempre he sido muy nerviosa; al estar en frente de alumnos, pero pienso que sí puedo enseñar".

E4: " "mi intención no sólo es ser un licenciado de educación media, mis planes es sacar una maestría, para po der trabajar a nivel superior; ¿por qué? Porque a nivel superior puedo contribuir a formar maestros con vocación".

E5: "Una porque he decido ser maestra, verdad". "El poder dar clases a nivel medio y yo aspiro no sólo la licenciatura sino que 
Pastor Umanzor

seguir a la maestría".

E20: "quiero ser un maestro de matemáticas, verdad, para transmitir mis co no cimientos a o tras personas y así tratar de que ellas les gusten más la clase".

En la visión de estos estudiantes existe una tendencia clara: con dificultades para aprender matemáticas vienen a la univer sidad a mejorar sus rendimientos académicos porque lo ven como un reto personal y profesional. Lo que esperan es que los profesores universitarios le permitan la construcción de los conocimientos sustantivos, sintácticos y sobre todo didácticos que los posicione donde ellos quieren.

\section{Tradición familiar}

E29: "Me gustan las matemáticas, esa es mi área, me gustan las matemáticas, y me ha gustado la docencia, tomar mi tiempo y explicarle a alguien para hacer otra cosa". "la matemática no sólo es práctica, lleva teoría, lleva lógica". "vine a la univer sidad a aprender un poco más".

\section{Conclusiones}

En consideración con los resultados obtenidos se puede concluir que:

1. Las creencias de los estudiantes por ser profesores de matemáticas están asociadas con el interés, dedicación y voluntad a las clases y al estudio, en función de lo que les enseñen los profesores. Esto implica que el docente universitario se percate de las diferencias en el moldeo de este estudiante, para lograr dos objetivos: el aprender matemáticas y aprender a enseñar matemáticas.

2. La identificación de los patrones simbólicos, numéricos y geométricos es posible gracias a la prueba y a la entrevista en profundidad asociada en las que participaron los 6 estudiantes, con el ánimo de establecer las tendencias en los patrones se llega al 
acuerdo que predominan los patrones numéricos, como era de esperarse. Ello implica una oportunidad de replantear el siguiente curso, en términos de una asociación en el desarrollo del pensamiento matemático y el de ser profesor de matemáticas.

3. Las competencias matemáticas identificadas en los estudiantes reflejan posibilidades de comprensión y razonamiento de 4 estudiantes, y solamente dos tienen las características que les permite hasta establecer metas a corto, mediano y largo plazo. Esto potencia a estos dos últimos estudiantes para arribar a un desar rollo de un pensamiento matemático sostenido. Ello exige una postura de los profesores más centrada en la ciencia matemática y no en su operatoria ni en lo utilitario de las matemáticas.

4. Los estudiantes entrevistados muestran las competencias: comprensión, razonamiento, aprendizaje y cognición definidas por el C N B, para la educación media, por tanto, se aceptaría que tienen potencialidades para el alcance de un desarrollo sostenido del pensamiento matemático, pero hace falta un trabajo sistemático en los niveles precedentes para aprender y enseñar matemáticas.

5. La identificación de patrones matemáticos (simbólicos, numéricos y geométricos) de manera conjunta en el primer ejercicio es el lo gro más importante de los estudiantes evaluados y entrevistados. Condición que da lugar al surgimiento de las competencias matemáticas (comprensión, razonamiento, aprendizaje y co gnición) que garantiza el alcance de un pensamiento matemático sostenido, que es lo que cubre la tesis de los estudiantes: ser buenos profesores de matemáticas, aunque hayan partido de experiencias negativas previas. Están conscientes que su éxito profesional como profesores de matemáticas que enseñarán matemáticas con la actitud científica de profesores dependerá del enfoque de formación basada en competencias que propugna la UPNFM. Esto tiene como implicaciones, que los profesores de la UPNFM revisen sus concepciones sobre la enseñanza y sus prácticas pedagógicas, para formar docentes que saben enseñar matemáticas. 
Pastor Umanzor

\section{Recomendaciones}

1. Se espera que el docente universitario tome en cuenta que los estudiantes sean parte de un proceso de preparación que los lleve a lograr do s objetivos: el aprender matemáticas y aprender a enseñar matemáticas.

2. Se espera que el docente universitario pueda replantear cada curso en adelante, pues si ya se tiene un grupo de estudiantes de la especialidad, es el momento de ayudarles a aprender matemáticas como ciencia y procurar desarrollar el pensamiento matemático de ellos como la base de su formación como profesor de matemáticas.

3. Se espera que el docente universitar io asuma una po stura que defina la enseñanza de las matemáticas como ciencia fundamentalmente y ello dé paso a la adopción de una actitud científica, necesaria en la construcción de una sociedad informada.

4. El docente universitario podría asumir la decisión de preguntarse, individual y colectivamente, sobre la posibilidad de enseñar las matemáticas como ciencia, pues así lo exige el proceso de acreditación internacional a la que está expuesta, ahora la carrera de matemáticas.

5. Los profesores de la UPN FM podrían revisar sus concepciones sobre la enseñanza y sus prácticas pedagógicas, para que los estudiantes aprendan matemáticas y aprendan a enseñar matemáticas, a partir de procesos inductivos, deductivos y su combinación, mismos que inicien con la identificación de patrones matemáticos que los lleve a desarrollar competencias matemáticas específicas y les permita a los estudiantes a alcanzar un pensamiento matemático claro como la garantía de mejorar la enseñanza de las matemáticas en el país. 
Patrones, competencias y desarrollo del pensamiento matemático

\section{Referencias Bibliográficas}

Barnett, Ronald (1998). Los límites de las competencias. La educación superior, el conocimiento y la sociedad. Editorial G edisa. España.

Cantoral, Ricardo (2002). Enseñanza de la M atemática en la Educación Superior. México: En Revista Sinéctica 19, Cátedra.D isponible en http:/ Lportal.iteso.mx/portal/page/portal/Sinectica/Historico/ N umeros anteriores04/019/19\%20Ricardo\%20C antoral-C atedra.pdf, consultado el 180414.

Godino, Juan D. (1991). Hacia unaTeoríia de la Didáctica de la M atemática. Madrid: En:A. Gutiérrez (Ed.), Área de Conocimiento: D idáctica de la Matemática (pp. 105-148) Síntesis. Disponible en http:// webdelpro fesor.ula.ve/nucleo tachir a/o scarg/materias/materias/ didactica/lecturas/temal.godino haciatem.pdf, consultado el 040114.

Kilpatrick, Jeremy (1998). Investigación en educación matemática: su historia y algunos temas de actualidad. USA: U niversidad de Georgia. Disponible en http://funes.uniandes.edu.co/679/1/ KilpatrickEducacion.pdf\#page=11, consultado el 030114.

N agusia, Berrítzegune (2014). Competencia matemática. Educación Secundaria Obligatoria. España: Universidades e Investigación. D isponible en http://www.hezkuntza.ejgv.euskadi.net/r43-2459/es/contenidos/ informacion/dig_publicaciones_innovacion/es_curricul/adjuntos/ 14 curriculum competeñcias_300l 300011c_Pub_BN_Competencia_Mate_ESO_c.pdf, consultado el 180414.

Polya, George (1987). Cómo plantear y resolver un problema. Editorial Trillas, México.

Senechal, Marjorie (2004). Forma. En Steen, Lynn A. (Editor) (2004). La enseñanza agradable de las matemáticas. México: Editorial LIMUSA, Traducción de Rodolfo Piña García. 
Pastor Umanzor

Steen, Lynn A. (2004). Patrones. En Steen, Lynn A. (Editor) (2004). La enseñanza agradable de las matemáticas. México: Editorial LIMUSA, Traducción de Rodolfo Piña García.

Trinidad,A ntonio; C arrero,Virginia;y, Soriano, Rosa M aría (2006). Teoría Fundamentada. La construcción de la teoría a través del análisis interpretacional. España: Centro de Investigaciones Sociológicas, C uadernos Metodológicos, $N^{\circ} 37$.

Tobón, Sergio (2000). La formación basada en competencias. 